\title{
Electrical Performance Enhancement of Al-Zn-Sn-O Thin Film Transistor by Supercritical Fluid Treatment
}

\author{
Li-Feng Teng, Po-Tsun Liu, Senior Member, IEEE, and Wei-Ya Wang
}

\begin{abstract}
In this letter, a low-temperature supercritical fluid (SCF) treatment was employed to enhance the electrical and optical properties of amorphous Al-Zn-Sn-O thin film transistors (a-AZTO TFTs) for flat-panel displays. The carrier mobility and threshold voltage of a-AZTO TFT were improved significantly after SCF process because of the reduction of trap density in the a-AZTO active layer. In addition, the SCF-treated a-AZTO TFT exhibited superior electrical reliability and less degradation after negative gate bias illumination stress. X-ray photoelectron spectroscopy analysis confirmed that the proposed SCF treatment could effectively oxidize a-AZTO film and change the oxidation states of $\mathrm{Sn}$, resulting in the improvement of a-AZTO TFT device characteristics.
\end{abstract}

Index Terms- Al-Zn-Sn-O thin film transistor (Al-Zn-Sn-O TFT), supercritical fluid (SCF), transparent amorphous oxide semiconductor (TAOS).

\section{INTRODUCTION}

$\mathbf{T}$ HIN film transistors (TFTs) using transparent amorphous oxide semiconductor (TAOS) have attracted great attentions for the backplane technology of a large-sized liquidcrystal displays and active matrix organic light-emitting diode displays because of its superior characteristics of high mobility, room temperature deposited, good uniformity, and compatibility with present display industry [1]-[3]. Transparent metal oxide TFTs using In-Zn-O [4], Hf-In-Zn-O [5], $\mathrm{In}-\mathrm{Zn}-\mathrm{Sn}-\mathrm{O}$ [6], and $\mathrm{In}-\mathrm{Ga}-\mathrm{Zn}-\mathrm{O}$ [7] as an active channel layer have been widely studied in the recent years. Although those TFTs performed good electrical performance, containing the rare-dispersive elements will be a critical issue for the longterm applications [8]. Therefore, rare elements-free transparent metal oxide semiconductors are considered to be the promising candidates for the next generation display technologies [9]. In this letter, a TAOS material, Al-Zn-Sn-O (AZTO), is employed as active channel layer of TFTs. All components in AZTO are plenty and universal in the earth. Some previous literatures reported AZTO active layer could be deposited at room temperature and the TFTs exhibit prominent electrical

Manuscript received June 18, 2013; revised June 30, 2013; accepted June 30, 2013. Date of publication August 2, 2013; date of current version August 21, 2013. This work was supported by the National Science Council of Taiwan under Contract NSC 100-2628-E-009-016-MY3. The review of this letter was arranged by Editor K.-S. Chang-Liao.

L.-F. Teng is with the Department of Photonics and Institute of ElectroOptical Engineering, National Chiao Tung University, Hsin-Chu 30010, Taiwan.

P.-T. Liu and W.-Y. Wang are with the Department of Photonics and Display Institute, National Chiao Tung University, Hsin-Chu 30010, Taiwan (e-mail: ptliu@mail.nctu.edu.tw).

Digital Object Identifier 10.1109/LED.2013.2272117 performance [10]. In this letter, a supercritical fluid (SCF) technology is proposed at $150{ }^{\circ} \mathrm{C}$ to enhance the electrical performance and reliability of AZTO TFTs. The SCF provides good liquid-like solvency and high gas-like diffusivity, giving it excellent transport capacity [11], [12]. The oxidant is also easily dissolved in SCF with specific surfactants. It is thereby allowed for the SCF to transport the oxidant and penetrates the active layer for trap passivation and interface oxidation at low temperature for promoting the electrical characteristics of AZTO TFT. Photoreaction of the SCF-treated AZTO TFT also is discussed to further examine the potential of AZTO TFT for flat-panel display backplane technologies.

\section{DEVICE FABRICATION}

TFT devices were fabricated on the silicon wafer with a bottom-gated inverted-staggered structure. First, a 100-nmthick thermal oxide was thermally grown on $\mathrm{n}^{+}$heavily doped (100) silicon substrate, which acted as a gate electrode. The active channel layer of a 25-nm-thick AZTO layer was formed by RF reactive sputtering with a power of $80 \mathrm{~W}$ at room temperature in a mixture of argon and oxygen with target of 3:67:30 mol\% $\left(\mathrm{Al}_{2} \mathrm{O}_{3}: \mathrm{ZnO}: \mathrm{SnO}_{2}\right)$. The flow rates of argon and oxygen gases were 10 and 2 SCCM, respectively. Then, a 100-nm-thick indium tin oxide was formed serving as source/drain electrodes by RF sputtering system and all the layers were defined by shadow masks. Both the channel width and length of AZTO TFTs were varied from 1000 to $200 \mu \mathrm{m}$. Sequentially, all samples were thermally annealed at $450{ }^{\circ} \mathrm{C}$ for $1 \mathrm{~h}$ in a furnace with $\mathrm{N}_{2}$ gas flow rate of $10 \mathrm{~L} / \mathrm{h}$ under atmosphere pressure, and denoted as the standard samples. It was followed that the samples were posttreated and divided into two groups for study in this letter. In the first group, the sample was placed in a SCF system at $150{ }^{\circ} \mathrm{C}$ for $1 \mathrm{~h}$, where it was injected with 2000-3000 psi of supercritical $\mathrm{CO}_{2}\left(\mathrm{SCCO}_{2}\right)$ fluid that were mixed with $5 \mathrm{vol} \%$ of propyl alcohol and 5 vol\% of pure $\mathrm{H}_{2} \mathrm{O}$. The propyl alcohol acts as a surfactant between nonpolar- $\mathrm{SCCO}_{2}$ fluid and polar$\mathrm{H}_{2} \mathrm{O}$ molecules, such that the $\mathrm{H}_{2} \mathrm{O}$ molecules are uniformly distributed in supercritical $\mathrm{CO}_{2}$ fluid. The $\mathrm{SCCO}_{2}$ fluid acted as a transporter to carry the $\mathrm{H}_{2} \mathrm{O}$ molecules into metal oxide films [13]. In the second group, samples were immersed in pure $\mathrm{H}_{2} \mathrm{O}$ vapor at $150{ }^{\circ} \mathrm{C}$ for $1 \mathrm{~h}$ for comparison to clarify the effects of $\mathrm{H}_{2} \mathrm{O}$ vapor. All electrical measurements were carried out by using the semiconductor parameter analyzer, Keithley 4200. The material analysis of X-ray photoelectron 

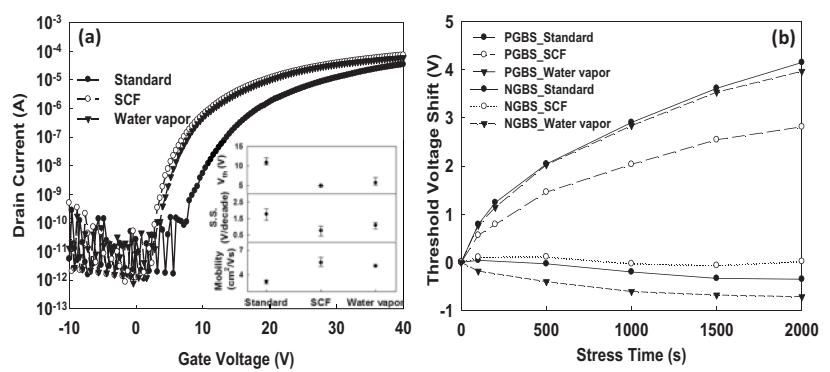

Fig. 1. (a) Drain current $\left(\mathrm{I}_{D}\right)$ versus gate voltage $\left(\mathrm{V}_{G}\right)$ curves of a-AZTO TFT devices with different posttreatments at a drain-to-source voltage of $11 \mathrm{~V}$. Inset: device parameters, including threshold voltage $\left(\mathrm{V}_{\text {th }}\right)$, subthreshold swing (SS), and mobility $(\mu)$. (b) Threshold voltage shifts of a-AZTO TFT devices with different posttreatments as a function of gate bias stress. The conditions of GBS test were under the positive and negative gate bias with an electrical field of $2.5 \mathrm{MV} / \mathrm{cm}$ and source/drain were grounded for $2000 \mathrm{~s}$.
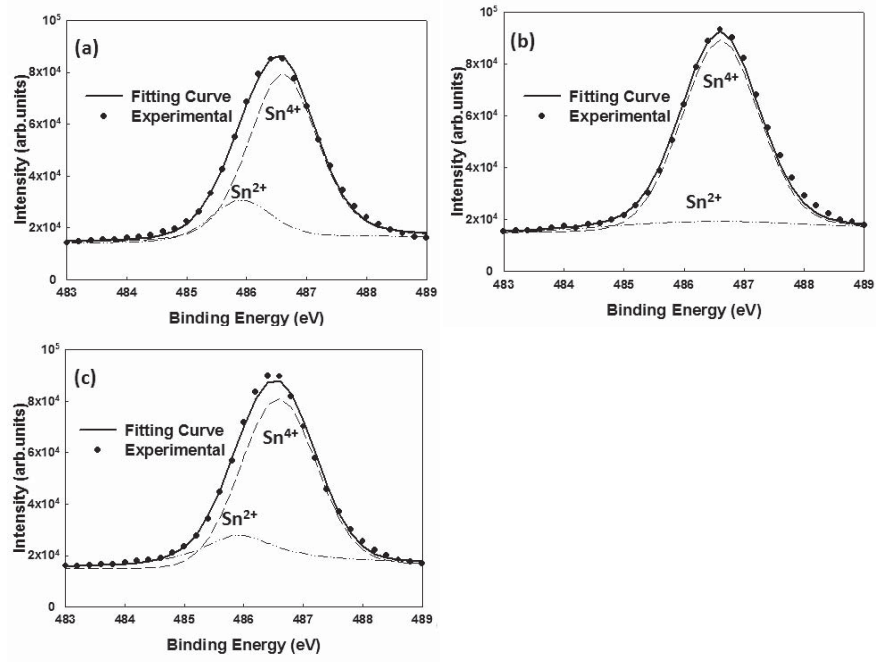

Fig. 2. XPS analysis of $\mathrm{Sn} 3 \mathrm{~d}_{5 / 2}$ spectrum for the a-AZTO thin films after (a) standard process, (b) SCF posttreatment, and (c) water vapor treatment.

spectroscopy (XPS) was also performed to examine the evolution of chemical bondings for $25 \mathrm{~nm}$-thick AZTO thin films with different posttreatments.

\section{RESULTS AND DISCUSSION}

Fig. 1(a) shows the transfer characteristics of AZTO TFTs with different posttreatments. The drain-to-source voltage $\left(V_{\mathrm{DS}}\right)$ was $11 \mathrm{~V}$. The extracted threshold voltage $\left(V_{\mathrm{th}}\right)$, subthreshold swing (SS), and carrier mobility of each AZTO TFT are also compared in the inset. The mobility was extracted by the slope of $\left|I_{D}\right|^{1 / 2}-V_{G}$ plot as equation, $\mu=2 L / W C_{\mathrm{OX}}\left(\partial\left|I_{D}\right|^{1 / 2} / \partial V_{G}\right)^{2}$. It is clearly observed that after SCF process, the threshold voltage of AZTO TFT decreased from 10.84 to $4.93 \mathrm{~V}$, and the value of SS also was improved from 1.76 to $0.77 \mathrm{~V} /$ decade. In addition, the mobility was enhanced significantly from 3.16 to $5.51 \mathrm{~cm}^{2} / \mathrm{Vs}$. Trap densities $\left(N_{t}\right)$ can be estimated from SS value with the following formula. $\mathrm{SS}=\log _{\mathrm{e}} 10 \times\left(k_{B} T / e\right)\left[1+e\left(t N_{t}+D_{\mathrm{it}}\right) / C_{i}\right]$, where $k_{\mathrm{B}}$ is the Boltzmann constant, $T$ is the temperature, $e$ is the elementary electric charge, $t$ is the thickness of the AZTO active layer, and $D_{\text {it }}$ is the interface trap density, assuming the trap $t N_{\mathrm{t}}$ dominated and $D_{\mathrm{it}}$ is negligible [14], [15].
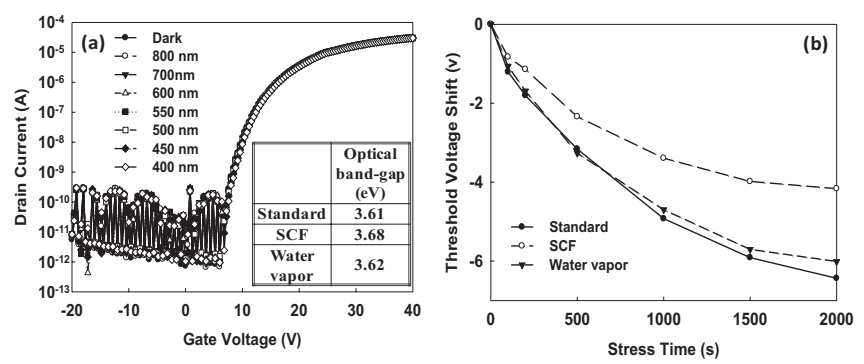

Fig. 3. (a) Transfer characteristics of the a-AZTO TFTs without any posttreatments under light radiation. Inset: corresponding values of optical energy bandgap $\left(\mathrm{E}_{g}\right)$ of a-AZTO films after different posttreatments. (b) Threshold voltage shifts of a-AZTO TFT devices with different posttreatments as a function of negative GBS under light illumination.

The $N_{\mathrm{t}}$ value of a-AZTO TFT for the standard, SCF-treated, and the water vapor process was $1.02 \times 10^{18}, 2.48 \times 10^{18}$, and $1.49 \times 10^{18} \mathrm{~cm}^{-3}$, respectively. This indicated that the proposed SCF treatment effectively decreased the trap density of AZTO active layer and improved the electrical performance of AZTO TFTs. In contrast, the performance of AZTO TFTs with $\mathrm{H}_{2} \mathrm{O}$ vapor treatment was also improved. Chen et al. [11] reported that the $\mathrm{H}_{2} \mathrm{O}$ absorbed in metal oxide thin films was dissociated into $\mathrm{H}$ and $\mathrm{OH}$ groups. The hydrogen atoms could be incorporated into metal oxide thin film to form hydrogen-related donors, whereas the dangling bonds or traps in the metal oxide thin film could be passivated by $\mathrm{OH}$ and form $\mathrm{M}-\mathrm{OH}$ bonds ( $\mathrm{M}$ is a metal cation). The passivation effect of $\mathrm{OH}$ on the amorphous AZTO film with large amount of dangling bonds will be more significant. In addition, Nomura et al. [14] reported that the $\mathrm{OH}$ bonds in metal oxide film would react with neighboring hydrogen and generate $\mathrm{H}_{2} \mathrm{O}$ molecules by the following equation: $\mathrm{M}-\mathrm{OH}+\mathrm{M}-\mathrm{OH} \rightarrow$ $\mathrm{V}_{\ddot{\mathrm{o}}}+\mathrm{M}-\mathrm{O}-\mathrm{M}+\mathrm{H}_{2} \mathrm{O} \uparrow+2 \mathrm{e}^{-}$, where $\mathrm{M}$ is a metal cation and $V_{\ddot{o}}$ is an ionized oxygen vacancy. It was also consisted with the experiment results, both SCF and $\mathrm{H}_{2} \mathrm{O}$ vapor could improve the electrical performance of AZTO TFTs. The results of gate bias stress (GBS) are shown in Fig. 1(b). In this measurement, an electric field of $2.5 \mathrm{MV} / \mathrm{cm}$ was applied to gate electrode and source/drain electrodes were grounded. The instability of SCF treated a-AZTO TFT was improved effectively, and the value of $V_{\text {th }}$ shift after positive GBS testing was $2.81 \mathrm{~V}$, whereas it was $4.15 \mathrm{~V}$ for the a-AZTO TFT without SCF treatment. Comparatively, the improvement in $V_{\text {th }}$ shift was not observed for the $\mathrm{H}_{2} \mathrm{O}$ vapor-treated one. It indicated that the effects of SCF treatment were different from the $\mathrm{H}_{2} \mathrm{O}$ vapor treatment. The XPS analysis was used further to identify the physical mechanism for electrical enhancement of a-AZTO TFTs. Fig. 2 shows the XPS of $S n 3 d_{5 / 2}$ spectrum for the a-AZTO thin film with different posttreatments. The peak of $\mathrm{Sn} 3 \mathrm{~d}_{5 / 2}$ signal has two components with binding energies of 486.6 and $485.9 \mathrm{eV}$, respectively, which were fitted by GaussianLorentzian deconvolution. The higher binding energy centered at 486.6 is originated from the $\mathrm{Sn}^{4+}$ bonding state, whereas the lower binding energy centered at 485.9 is attributed to the $\mathrm{Sn}^{2+}$ binding state [16]. The ratio of $\mathrm{Sn}^{4+}$ component, which was defined as $\mathrm{Sn}^{4+} /\left(\mathrm{Sn}^{4+}+\mathrm{Sn}^{2+}\right)$ were $81.1 \%$, $89.1 \%$, and $82.6 \%$ for the a-AZTO film with the standard 
process, SCF treatment, and $\mathrm{H}_{2} \mathrm{O}$ treatment, respectively. The performance of a-AZTO TFTs was strongly dominated by $\mathrm{Sn}^{4+}$, because the field-effect carrier mobility of amorphous oxides was controlled by heavy metal cations with $(n-1) d^{10} n s^{0}(n \geq 4)$ electronic configurations. In the a-AZTO thin film, $\mathrm{Sn}^{4+}$ with the electron configuration of $4 \mathrm{~d}^{10} 5 \mathrm{~s}^{0}$ dominated the mobility owing to $5 \mathrm{~s}^{0}$ orbital of $\mathrm{Sn}^{4+}$ being larger than $4 \mathrm{~s}^{0}$ orbital of $\mathrm{Zn}^{2+}$ [10]. After SCF treatment, the ratio of $\mathrm{Sn}^{4+}$ increased from initial $81.1 \%$ to $89.1 \%$. This indicated SCF process could effectively oxidize the a-AZTO thin film and transform $\mathrm{Sn}^{2+}$ to $\mathrm{Sn}^{4+}$. The $\mathrm{SCCO}_{2}$ fluid owns superior capability to transport $\mathrm{H}_{2} \mathrm{O}$ molecules into metal oxide film, even arriving the interface and causing oxidation reaction [13]. This treatment has superior ability for oxidation and changes the $\mathrm{Sn}^{2+}$ into $\mathrm{Sn}^{4+}$. The improvement of interface could explain the enhancement of GBS test. The modification on the oxidation state of Sn ion enhanced the electrical performance and stability of a-AZTO TFTs. The electrical characteristics of standard a-AZTO TFTs under light radiation with wavelengths ranging from 800 to $400 \mathrm{~nm}$ were studied. Fig. 3(a) shows the $V_{\text {th }}$ value kept intact under visible-light illumination. The same phenomenon was also observed for the case of a-AZTO TFTs with SCF process or $\mathrm{H}_{2} \mathrm{O}$ vapor treatments (not shown). This inset of Fig. 3(a) extracted the optical band gap $\left(E_{\mathrm{g}}\right)$ of a-AZTO films with different posttreatments by using Tauc's model [17]. All the a-AZTO thin films performed $E_{\mathrm{g}}$ larger than $3.62 \mathrm{eV}$, explaining that electrical characteristics of a-AZTO TFTs were not affected by visible-light illumination because of the large band gap of active layer. Therefore, a-AZTO films would not easily absorb the light with wavelengths ranging from 800 to $400 \mathrm{~nm}$. The negative gate-bias illumination stress (NGBIS) was also examined further for the consideration of TFT-backplane technology as shown in Fig. 3(b). In the stress testing, source/drain electrodes were grounded and $25 \mathrm{~V}$ was applied to gate electrode. The light illumination with the intensity of $740 \mathrm{~mW} / \mathrm{cm}^{2}$ and wavelength of $546 \mathrm{~nm}$ was employed as radiation source. The $V_{\text {th }}$ shift of the SCF-treated a-AZTO TFT was only $-4.17 \mathrm{~V}$, whereas it was -6.44 and $-6.01 \mathrm{~V}$ for the standard and $\mathrm{H}_{2} \mathrm{O}$-vapor treated ones, respectively. Although the a-AZTO layer has no optical absorption at $546 \mathrm{~nm}$, the light illumination would effectively excite the oxygen vacancies $\left(V_{\mathrm{o}}\right)$ to become the charged oxygen vacancy $\left(\mathrm{V}_{\mathrm{o}}^{2+}\right)$ [18]. The generated $\mathrm{V}_{\mathrm{o}}^{2+}$ states act as the positive fixed charges, which were trapped at the interface by negative gate bias. Therefore, the effective gate bias was changed by those trapped charges resulting in a negative shift in transfer characteristics of a-AZTO TFT. The $V_{\text {th }}$ shift of SCF-treated a-AZTO TFT after NGBIS for $2000 \mathrm{~s}$ was less than the others because of the less trap density in the active a-AZTO layer. These above results indicated that the SCF process not only improved the electrical performance and stability of a-ATZO TFTs, but also reduced the electrical degradation after negative gate bias illumination stress.

\section{CONCLUSION}

In summary, this letter has demonstrated the effects of lowtemperature SCF on the a-AZTO TFTs. The SCF process could effectively oxidize the a-AZTO thin film and modify the oxidation states of $\mathrm{Sn}$, resulting in the improvement of electrical performance and stability of a-AZTO TFTs. In addition, the optical band gap increased after SCF process. After NGBIS, the $V_{\text {th }}$ shift of the SCF treated a-AZTO TFT was reduced significantly than the one without SCF posttreatment. Owing to its low process temperature and efficient trap passivation in active layer, the SCF posttreatment is highly promising for emerging amorphous oxide TFT manufacturing.

\section{REFERENCES}

[1] K. Nomura, H. Ohta, A. Takagi, et al., "Room-temperature fabrication of transparent flexible thin-film transistor using amorphous oxide semiconductors," Nature, vol. 432, no. 7016, pp. 488-492, Nov. 2004.

[2] D. Geng, D. H. Kang, M. J. Seok, et al., "High-speed and low-voltagedriven shift register with self-aligned coplanar a-IGZO TFTs," IEEE Electron Device Lett., vol. 33, no. 7, pp. 1012-1014, Jul. 2012.

[3] J. S. Park, W. J. Maeng, H. S. Kim, et al., "Review of recent developments in amorphous oxide semiconductor thin-film transistor devices," Thin Solid Films, vol. 520, no. 6, pp. 1679-1693, Jan. 2012.

[4] P. T. Liu, Y. T. Chou, and L. F. Teng, "Environment-dependent metastability of passivation-free indium zinc oxide thin film transistor after gate bias stress," Appl. Phys. Lett., vol. 95, no. 23, pp. 233504-1-233504-3, Dec. 2009.

[5] J. S. Park, T. S. Kim, K. S. Son, et al., "High-performance and stable transparent $\mathrm{Hf}-\mathrm{In}-\mathrm{Zn}-\mathrm{O}$ thin-film transistors with a double-etch-stopper layer," IEEE Electron Device Lett., vol. 31, no. 11, pp. 1248-1250, Nov. 2010.

[6] B. J. Kim, H. J. Kim, T. S. Yoon, et al., "Effects on annealing temperature for solution-processed IZTO TFTs by nitrogen incorporation," Electrochem. Solid-State Lett., vol. 13, no. 12, pp. H419-H422, Sep. 2010.

[7] J. S. Lee, S. Chang, S. M. Koo, et al., "High-performance a-IGZO TFT With $\mathrm{Zr}_{2} \mathrm{O}_{5}$ gate dielectric fabricated at room temperature," IEEE Electron Device Lett., vol. 31, no. 3, pp. 225-227, Mar. 2010.

[8] Y. S. Rim, D. L. Kim, W. H. Jeong, et al., "Effect of $\mathrm{Zr}$ addition on $\mathrm{ZnSnO}$ thin-film transistors using a solution process," Appl. Phys. Lett., vol. 97, no. 23, pp. 233502-1-233502-3, Dec. 2010.

[9] P. K. Nayak, J. V. Pinto, G. Goncalves, et al., "Environmental, optical, and electrical stability study of solution-processed Zinc-Tin-Oxide thinfilm transistors," J. Display Technol., vol. 7, no. 12, pp. 640-643, Dec. 2011.

[10] D. H. Cho, S. Yang, C. Byun, et al., "Transparent Al-Zn-Sn-O thin film transistors prepared at low temperature," Appl. Phys. Lett., vol. 93, no. 14, pp. 142111-1-142111-3, Oct. 2008.

[11] M. C. Chen, T. C. Chang, S. Y. Huang, et al., "A low-temperature method for improving the performance of sputter-deposited $\mathrm{ZnO}$ thinfilm transistors with supercritical fluid," Appl. Phys. Lett., vol. 94, no. 16, pp. 162111-1-162111-3, Apr. 2009.

[12] P. T. Liu, C. T. Tsai, T. C. Chang, et al., "Effects of supercritical fluids activation on carbon nanotube field emitters," IEEE Trans. Nanotechnol., vol. 6, no. 1, pp. 29-34, Jan. 2007.

[13] P. T. Liu, C. T. Tsai, and P. Y. Yang, "Effects of supercritical $\mathrm{CO}_{2}$ fluid on sputter-deposited hafnium oxide," Appl. Phys. Lett., vol. 90, no. 22, pp. 223101-1-223101-3, May 2007.

[14] K. Nomura, T. Kamiya, H. Ohta, et al., "Defect passivation and homogenization of amorphous oxide thin-film transistor by wet $\mathrm{O}_{2}$ annealing," Appl. Phys. Lett., vol. 93, no. 19, pp. 192107-1-192107-3, Nov. 2008.

[15] L. F. Teng, P. T. Liu, Y. J. Lo, et al., "Effects of microwave annealing on electrical enhancement of amorphous oxide semiconductor thin film transistor," Appl. Phys. Lett., vol. 101, no. 13, pp. 132901-1-132901-4, Sep. 2012.

[16] M. Kwoka, L. Ottaviano, M. Passacantando, et al., "XPS study of the surface chemistry of L-CVD $\mathrm{SnO}_{2}$ thin films after oxidation," Thin Solid Films, vol. 490, no. 1, pp. 36-42, Oct. 2005.

[17] D. L. Wood and J. Tauc, "Weak absorption tails in amorphous semiconductors," Phys. Rev. B, vol. 5, no. 8, pp. 3144-3151, Apr. 1972

[18] H. Oh, S. M. Yoon, M. K. Ryu, et al., "Photo-accelerated negative bias instability involving subgap states creation in amorphous In-Ga-Zn-O thin film transistor," Appl. Phys. Lett., vol. 97, no. 18, pp. 183502-1-183502-3, Nov. 2010. 